Conclusion Short-term use of CT-1C-terminal peptide early in reperfusion can reduce myocardial tissue injury and oxidative damage, as well as the extent of cardiomyocyte apoptosis, so that the extension of animal survival time; but the intraperitoneal injection of CT-1C-terminal peptide after a longer period of time reduced the tolerance of SD rats on ischaemia reperfusion injury, the tissue injury and the extent of oxidative damage increased significantly, and cardiac myocyte apoptosis have occurred in the surrounding area of infarction, and the animals have a shorter survival time.

\section{e0123 THE EFFECT OF DIABETES ON PROTECTION OF ISCHAEMIC POSTCONDITIONING IN MYOCARDIAL ISCHAEMIA- REPERFUSION INJURY}

doi:10.1136/hrt.2010.208967.123

Zhao Xin, Yu Xuefan, Quan Nanhu. Department of Cardiology, First Hospital of Jilin University, Changchun, China

Objective Study on the effect of diabetes on protection of ischaemia Postconditioning in myocardial ischaemia-reperfusion injury in isolated rat hearts.

Methods The type 2 diabetic rats were induced by the intravenous injection of streptozotocin (STZ) and high caloric diet. 60 Wister rats were divided into three groups randomly. Ischaemia- reperfusion in nomal rats (A group), ischaemia postconditioning in nomal rats (B group), ischaemia postconditioning in diabetic rats ( $\mathrm{C}$ group). Rats were used for Langendorff isolated heart perfusion with $30 \mathrm{~min}$ of globe ischaemia and $60 \mathrm{~min}$ of reperfusion, then the models of Ischaemia- reperfusion (A) were made. But to B and C, rat hearts were subjected to six cycles of $10 \mathrm{~s}$ of globe ischaemia and $10 \mathrm{~s}$ of reperfusion as ischaemia postconditioning during the early minutes of reperfusion. The levels of lactate dehydrogenase $(\mathrm{LDH})$ in the coronary effluent and infarction size was determined by TTC staining. Phosphorylation of akt and gsk- $3 \beta$ were analysed by western blotting and immunohistochemical staining.

Results Ischemic postconditioning reduced $\mathrm{LDH}, \mathrm{CK}$ and improved the haemodynamic parameters and reduced myocardial infarction size $(29.50 \pm 3.4 \%$ vs $45.65 \pm 4.8 \%$ ), phospho-Akt and phospho-GSK$3 \beta$ expression increased markedly in B group. But compared A group there were no parently diffrence in $\mathrm{C}$ group. The level of $\mathrm{LDH}, \mathrm{CK}$ didn't decline and the myocardial infarction size were not reduced. phospho-Akt and phospho-GSK-3 $\beta$ expression in C group is more less than in B group.

Conclusion Ischemic postconditioning may significantly protect myocardium from reperfusion injury in isolated normal rat hearts. But in diabetic rats, the protection of Ischaemic postconditioning has no effect, the mechanism of this phenomenon maybe connected with lower expression of Phosphorylation of Akt and GSK-3 $\beta$ in the condition of diabetic and impaired Reperfusion Injury Salvage Kinase (RISK) signalling pathway (RISK pathway).

\section{e0124 EFFECTS OF OXIDATIVE STRESS AND GENDER DIFFERENCES IN SD RATS WITH HIGH-SALT HYPERTENSION VIA ACUTE SHORT-TERM COLD EXPOSURE}

doi:10.1136/hrt.2010.208967.124

Lei Guangtao, Wu Qinghua. Second Affiliated Hospital of Nanchang University, Nanchang, China

Objective To perform high-salt hypertension model in SD rats and observe effects and oxidative stress and gender differences in SD rats, and then evaluate mechanism that blood pressure is affected by oxidative stress induced by cold environment.

Methods The male and female SD rats were randomly divided into four groups $(n=8)$ : male control group (MC), female control group
(FC), male high-salt group (MS), female high-salt group (FS), MC and FC were fed regularly, MS and FS were fed with diet composed of $8 \%$ salt. Feeding period was 8 weeks. Four groups were fed regularly in ninth week; in the first 10 weeks, four groups were put into a $4^{\circ} \mathrm{C}$ artificial climate box in tenth, $1 \mathrm{~h}$ per day. Systolic blood pressure (SBP) in SD rats was measured every other day from 1st week to in first 4 days 9 th week with tail cuff. Systolic blood pressure in SD rats was measured daily with tail cuff in late 3 days of 9 th week and 10th weeks.24-h urine in each group was collected by biological metabolism, calculated accurately.

Results 1. High-salt diet for 8 weeks, MS group and FS group blood pressure was significantly higher than the control group the same sex $(p<0.05)$. In 10th weekend four sets of blood pressure after cold exposure (BP) were higher, MS group and the FS group blood pressure $\triangle \mathrm{BP}(\triangle \mathrm{BP}=$ before exposure $\mathrm{BP}$-after exposure $\mathrm{BP})$ significantly higher than the control group the same sex $(p<0.01)$. 2. 8 week FS and MS $24 \mathrm{~h}$ urine volume, urinary MALB, urinary RBP, urine sodium, urine potassium excretion higher than that of the same sex control group $(p<0.01)$; MS and FS groups showed no change in exposure;3. After cold exposure high salt group $24 \mathrm{~h}$ urinary 8-isoPGF2 $\alpha$ excretion compared with before the cold exposure was significantly higher $(p<0.01)$, serum Ang II levels than before the cold increased and serum NO concentration decreased $(p<0.05)$, while no change in the control group. Before and after cold exposure the MS and FS, MC compared with FC no gender differences emerged. 4. After cold exposure $\mathrm{NADPH}$ oxidase activity and SOD activity, MS compared with FS, MC compared with FC does not appear gender differences, but the gender of the high salt group was significantly higher $(\mathrm{p}<0.05)$.

Conclusion 1. High-salt diet increased blood pressure, and high-salt diet on blood pressure after high salt gender differences emerged; resume normal diet of high salt hypertensive rats have a certain recovery of renal function, blood pressure, but high salt blood pressure, gender differences still exist in blood pressure. Control group with the same sex, short-term acute cold exposure for highsalt hypertensive rats blood pressure increased significantly; 2 . Cold high-salt hypertensive rats after exposure, oxidative stress increased; but male and female rats after exposure to cold and oxidative stress between the gender differences are not shown.

\section{e0125 MIMP-9 GENE POLYMORPHISMS CONTRIBUTE TO CORONARY ARTERY DISEASE RISK IN THE UIGHUR POPULATION OF CHINA}

doi:10.1136/hrt.2010.208967.125

Wang Lei, Ma Yitong, Yang Yining, Xie Xiang, Liu Fen. Department of Cardiovascular Medicine, The First Affiliated Hospital, Xinjiang Medical University, Urumqi, China

Background Matrix metalloproteinase-9 (MMP-9) plays a pivotal role in early atherosclerosis, vascular remodelling and development of atherosclerotic lesion. The potentially functional MMP-9 polymorphisms may contribute to the susceptibility of coronary artery disease (CAD). We aimed to investigate the association between three SNPs (-1562C > T, R279Q, R668Q) of the MMP-9 gene with $\mathrm{CAD}$ in the Uighur population of China.

Materials and methods 375 angiographic ally proven patients with coronary artery disease and 417 sex-matched and ethnically matched controls were genotyped for MMP-9 polymorphisms by the PCR-restriction fragment length polymorphism (PCR-RFLP) technique. Genotype/allele frequencies were compared in patients and controls using the $\chi^{2}$ test. The relationship between the polymorphism of the MMP-9 gene and the severity of coronary arterial stenosis was analysed also.

Results At MMP-9 -1562 locus, there were significant differences between patients and controls $(p<0.05)$, leading to significant $O R$ for TT genotype ( $\mathrm{OR}=2.93$, CI 1.03 to 8.72$)$ and $\mathrm{R}$ allele $(\mathrm{OR}=1.85$, 
CI 1.34 to 2.54). These OR were higher in the sub-sample of smokers (3.87 and 2.06, respectively). Binary logistic regression analysis also confirmed that $\mathrm{R}$ allele carriers (CT and TT) have a higher risk of $\mathrm{CAD}(\mathrm{OR}=2.07$, CI 1.09 to 2.95). MMP-9 R2790 locus did not show significant differences between patients and controls. But $\mathrm{QQ}$ genotype and $Q$ allele were significant risk factors in the smoker group. $\mathrm{Q}$ allele carriers ( $\mathrm{RQ}$ and $\mathrm{QQ}$ ) were also significantly associated with $\mathrm{CAD}$ risk in the smoker group $(\mathrm{OR}=1.43$, CI 1.13 to 1.2.26). The R6680 locus did not show significant differences between two groups. And the MMP-9 polymorphism may not be useful as a predictor of the severity of coronary atherosclerosis.

Conclusions MMP-9 -1562T allele and TT genotype are significantly associated with CAD patients from the Uighur Population of China (Xinjiang). This association was stronger in smokers, supporting the conclusion that an interaction between MMP-9 activity and smoking augments CAD risk. Further studies with larger sample size are warranted to confirm these associations in different populations.

\section{e0126 STUDY ON ANTI-OXIDATIVE FUNCTION OF FOUR KINDS OF SCHIZANDRAE LIGNANS}

doi:10.1136/hrt.2010.208967.126

Sun Xin, Xie Yu, Chen Jianguang, Wang Qi, Tian Dan, Li Tan. Life Science Center, Beihua University, Beihua, China

Objective To study the anti-oxidative function of schisandrin $\mathrm{A}$ (SinA), schisandrinB (SinB), schisandrolA (SolA) and schisandrin ester A (SesA).

Methods Using the method of the self oxidation method of pyrogallol, Fenton system.

Results The results shown that all of four kinds of schizandrae lignans have the inhibition function to Superoxide anion radical $\left(\mathrm{O}_{2}{ }^{-} \cdot\right)$, SinB had the highest inhibition rate which could arrive at $68.74 \%$; They also had the same inhibition to hydroxyl radical $(\mathrm{OH})$ and $\operatorname{Sin} B$ have the best effect.

Conclusions schisandrin A ( $\operatorname{Sin} A)$, schisandrin B ( $\operatorname{Sin} B)$, schisandrolA (SolA) and schisandrin ester A (SesA) can be used as a natural antioxidation for human cardiovascular disease treatment and preventive health care.

\section{e0127 DETERMINATION OF PULMONARY ARTERY PRESSURE AND CARDIAC OUTPUT IN RAT}

doi:10.1136/hrt.2010.208967.127

Ping Yuan, Rui Zhang, Dong Liu, Qianqian Liu. Shanghai Pulmonary Hospital, Tongji University, Shanghai, China

Objective To establish a method for determination of pulmonary artery pressure and cardiac output in rat.

Methods 20 Sprgue-Dawely rats were randomly assigned into two groups: control group and pulmonary arterial hypertension (PAH) group. Rats in $\mathrm{PAH}$ group were received a single subcutaneous injection of monocrotaline $(60 \mathrm{mg} / \mathrm{kg})$. The hand-made PE-50 catheters were inserted into pulmonary artery via right jugular vein, which we can perform mean pulmonary artery pressure. Similarly, cardiac output was detected through thermodilution method.

Results After 21 days, compared with control group, mean pulmonary artery pressure was significantly increased $(17.4 \pm 1.8 \mathrm{~mm} \mathrm{Hg}$ in control group vs $61.8 \pm 4.3 \mathrm{~mm} \mathrm{Hg}$ in $\mathrm{PAH}$ group, respectively) and cardiac output was significantly decreased $(130 \pm 5.8 \mathrm{ml} / \mathrm{min}$ in control group vs $71 \pm 6.7 \mathrm{ml} / \mathrm{min}$ in $\mathrm{PAH}$ group, respectively) in $\mathrm{PAH}$ group.

Conclusions This method is a simple and direct method to detect pulmonary artery pressure and cardiac output in rat.

\section{e0128 ANGIOTENSIN-(1-7) INHIBITS VASCULAR REMODELLING IN RAT JUGULAR VEIN GRAFTS VIA REDUCED ERK1/2 AND P38 MAPK ACTIVITY}

doi:10.1136/hrt.2010.208967.128

${ }^{1}$ Wu Jingguo, ${ }^{1}$ Liang Yanbing, ${ }^{1}$ Tang Hao, ${ }^{2}$ Tang Anli, ${ }^{1}$ Ma Zhongfu, ${ }^{1}$ Ma Hong. ${ }^{1}$ The First Affiliated Hospital Sun Yat-sen University/Department of General Internal Medicine, Guangzhou, China; ${ }^{2}$ The First Affiliated Hospital Sun Yat-sen University, Department of Cardiology, Guangzhou, China

Objects To evaluate the effects of Ang-(1-7) on vascular remodelling in vein grafts.

Methods a model of autologous jugular vein grafts in rats was established. With this model system, rats ( $n=12$ per group) underwent autologous jugular vein graft transplantation (Ang-(1-7) and control groups), or a sham operation (sham group) in which grafting was not performed. Three days after operation, minipumps were installed for continuous infusion of Ang-(1-7) $(25 \mu \mathrm{g} / \mathrm{kg} / \mathrm{h})$ or normal saline (control and sham groups) through the jugular vein. Results 4 weeks, weight, blood pressure and heart rate were not significantly different between groups. Typical venous-graft hyperplasia, vascular remodelling, ERK1/2 activity, p38 MAPK activity and proliferating cell nuclear antigen (PCNA) and a-smooth muscle actin (a-SMA) expression present in the control group were attenuated by continuous Ang-(1-7) infusion. Tissue angiotensin II expression was increased in the Ang-(1-7) and control groups but was not different between the groups.

Conclusion The results of the present study indicate that exogenous Ang-(1-7) interferes with the vascular remodelling of autologous jugular vein grafts and significantly inhibits vein-graft intimal hyperplasia via inhibition of vascular tissue ERK1/2 and p38 MAPK activation. Thus, exogenous Ang-(1-7) improves the outcome of vein grafting via attenuation of vascular remodelling.

\section{C0129 EFFECT OF TETRANDRINE ON ANOXIA/REOXYGENATION- INDUCED RELEASE OF PROINFLAMMATORY FACTORS IN CULTURED CARDIOCYTE OF NEONATE RATS}

doi:10.1136/hrt.2010.208967.129

Yuqin Wang, Yuqin Wang. The Pla 252 hospital

Objective To investigate the effect of tetrandrine on anoxia/reoxygenation-induced the release of myocardial enzyme $\mathrm{LDH}, \mathrm{CK}$ and proinflammatory factors: TNF- $\alpha$, IL- $1 \beta$, IL- 6 in cultured cardiocytes of neonate rates.

Methods After cardiocytes were cultured in vitro successfully, it were divided into four group: control group (CON), anoxia/reoxygenation group (A/R), tetrandrine group (Tet), simvastatin (Sim) in random. Each group was treated as follow: CON group - not treated anoxia/ reoxygenation, continuous incubated $26 \mathrm{~h}$ under normal circumstance. A/R group- first anoxia incubate carried, cells were incubated on the non- saccharide non- serum culture medium, which saturate by $95 \%$ argon gases $2 \mathrm{~h}$, reoxygenation incubate followed, cells were incubated in normal circumstance $24 \mathrm{~h} .0 .9 \%$ saline were added into culture fluid before the beginning of reoxygenation. Tet group and Sim group -the procedure of anoxia/reoxygenation was same to $\mathrm{A} / \mathrm{R}$ group, the difference of these two groups was they added Tet $(30 \mu \mathrm{mol} / \mathrm{l})$ or $\operatorname{Sim}(10 \mu \mathrm{mol} / \mathrm{l})$ respectively into culture fluid and incubated $60 \mathrm{~min}$ before anoxia beginning. LDH, CK, TNF- $\alpha$, IL-1 $\beta$, IL- 6 were detected after reoxygenation $24 \mathrm{~h}$.

Result The LDH and CK were increased significantly in $\mathrm{A} / \mathrm{R}$, Tet, and Sim groups compared with CON group $(p<0.01)$. The LDH and $\mathrm{CK}$ in Tet and Sim group were lower significant than $\mathrm{A} / \mathrm{R}$ group $(p<0.01) .2$. The proinflammatory factors TNF- $\alpha$, IL-1 $\beta$ and IL-6 were increased significantly in $A / R$, Tet, and Sim groups compared with CON group $(p<0.01)$. And it were lower significant than $A / R$ 